\title{
Contribuição para o Conhecimento da Fauna de Arctiinae (Lepidoptera: Erebidae) no Estado de Santa Catarina, Brasil
}

\author{
Janaína Madruga Silva ${ }^{\bowtie}$ Eduardo José Ely e Silva
}

Universidade Federal de Pelotas, e-mail: nina 0694@hotmail.com (Autor para correspondência ${ }^{\varpi}$ ), eduelysilva@uol.com.br.

\section{EntomoBrasilis 7 (3): 222-226 (2014)}

Resumo. Com o objetivo de contribuir para o conhecimento da fauna de Arctiinae (Lepidoptera: Erebidae) ocorrentes no Estado de Santa Catarina, elaborou-se uma lista das espécies depositadas no Museu Entomológico Ceslau Biezanko. Os dados são referentes a coletas realizadas na região entre os anos 1943 e 1976 por Ceslau Maria Biezanko, Vitor Becker e Fritz Plauman. Foram encontradas 101 espécies, distribuídas em 57 gêneros e 238 indivíduos de Arctiini. Um total de 15 espécies é novo registro para o estado.

Palavras-Chave: Arctiini; Mata Atlântica; Riqueza de Espécies.

\section{Contribution to the Knowledge of the Arctiinae (Lepidoptera: Erebidae) Fauna in the Santa Catarina State, Brazil}

Abstract. Aiming to contribute to the knowledge of the Arctiinae (Lepidoptera: Family: Erebidae) fauna occurring in the Santa Catarina State, drew up a list of species deposited in the Entomological Museum Ceslau Biezanko. The data relating to collections made in the region between 1943 and 1976 by Maria Ceslau Biezanko, Vitor Becker and Fritz Plauman. We found 101 species in 57 genera and 238 individuals of Arctiini. A total of 15 species are new record for the state.

Keywords: Arctiini; Atlantic Rainforest; Species Richness.

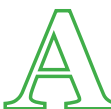
rctiinae é composta por um grupo diverso de mariposas de pequeno a médio porte que apresentam uma variedade enorme de formas e cores, incluindo espécies miméticas. Foi recentemente incluída como subfamília de Erebidae (Noctuidea), apresentando as tribos Arctiini, Lithosiini, Syntomini e Amerilini (ZAHIRI et al. 2011; ZAHIRI et al. 2012). Arctiinae possui aproximadamente 11 mil espécies cosmopolitas e seis mil na região Neotropical (HePPNER 1991). Para o Brasil são estimadas duas mil espécies (Brown JR. \& FreitAS 1999).

De acordo com Freitas et al. (2006), Arctiinae é um grupo bem distinto e moderadamente fácil de amostrar e identificar. Esta subfamília é bastante conhecida por estar entre os lepidópteros noturnos mais utilizados como bioindicadores em monitoramento de ecossistemas naturais (Hilty \& MERENLENdER 2000; Freitas et al. 2006). No sul do país, a fauna de arctíneos é relativamente bem estudada no estado do Rio Grande do Sul, com muitos trabalhos recentes (TESTON \& CoRseull 2002, 2003a, 2003b, 2004; TESTON et al. 2006; TESTON et al. 2009; FERRO \& ROMANOWSKI 2012).

No entanto, para Santa Catarina consta apenas uma lista de espécies baseada em compilação de dados de espécimes depositados em coleções brasileiras (FerRo et al. 2012). Devido ao pouco que se sabe sobre a fauna de Arctiinae no estado, o objetivo deste trabalho foi contribuir com a lista existente, através da adição de dados procedentes de coletas realizadas por Ceslau Maria Biezanko, Vitor Becker e Fritz Plaumann entre os anos de 1943 e 1976.

\section{MATERIAL E MÉTODOS}

A lista de espécies de Arctiinae ocorrentes no Estado de Santa Catarina foi elaborada a partir dos exemplares coletados por
Ceslau Maria Biezanko, Vitor Becker e Fritz Plaumann nas cidades de Blumenau, Joinville, Seara e Brusque (Figura 1), entre os anos de 1943 e 1976. O material analisado pertence ao Museu Entomológico Ceslau Biezanko da Universidade Federal de Pelotas (UFPel), Rio Grande do Sul, Brasil. Coordenadas geográficas, altitude, clima e tipo de vegetação de cada município estudado estão listados na Tabela 1.

Os exemplares estavam sendo mantidos em envelopes entomológicos, foram montados a seco e tiveram sua nomenclatura e sistemática confirmadas e atualizadas através da comparação com o material depositado no museu e a bibliografia especializada (HAMPSON 1898, 1900, 1901, 1915, 1920; SEITZ 1907; WATSON \& GOODGER 1986; ZAHIRI et al. 2012).

\section{RESULTADOS E DISCUSSÃO}

Foram listadas 101 espécies de Arctiini (Erebidae: Arctiinae) para o Estado de Santa Catarina, distribuidas em 57 gêneros e 238 indivíduos (Tabela 2). Arctiini tem sido relatada como predominante em muitos trabalhos (Hilt \& Fiedler 2006; Ferro \& Diniz 2007; FERro et al. 2012) dado que pode estar relacionado à grande diversidade da tribo, mas também ao maior interesse dos pesquisadores, visto ser um grupo bastante notável. De acordo com o material revisado, o município com maior número de espécies foi Blumenau (54), seguido de Seara (22), Joinville (14) e Brusque (07). Blumenau também apresentou o maior número de novos registros municipais (48), seguido de Seara (22), Brusque (04) e Joinville (02).

São novos registros para o estado, 15 espécies: Amaxia flavipuncta Hampson, 1904; Bertholdia soror Dyar, 1901; Cosmosoma ignidorsia Hampson, 1898; Dysschema lucifer Butler, 1873; 


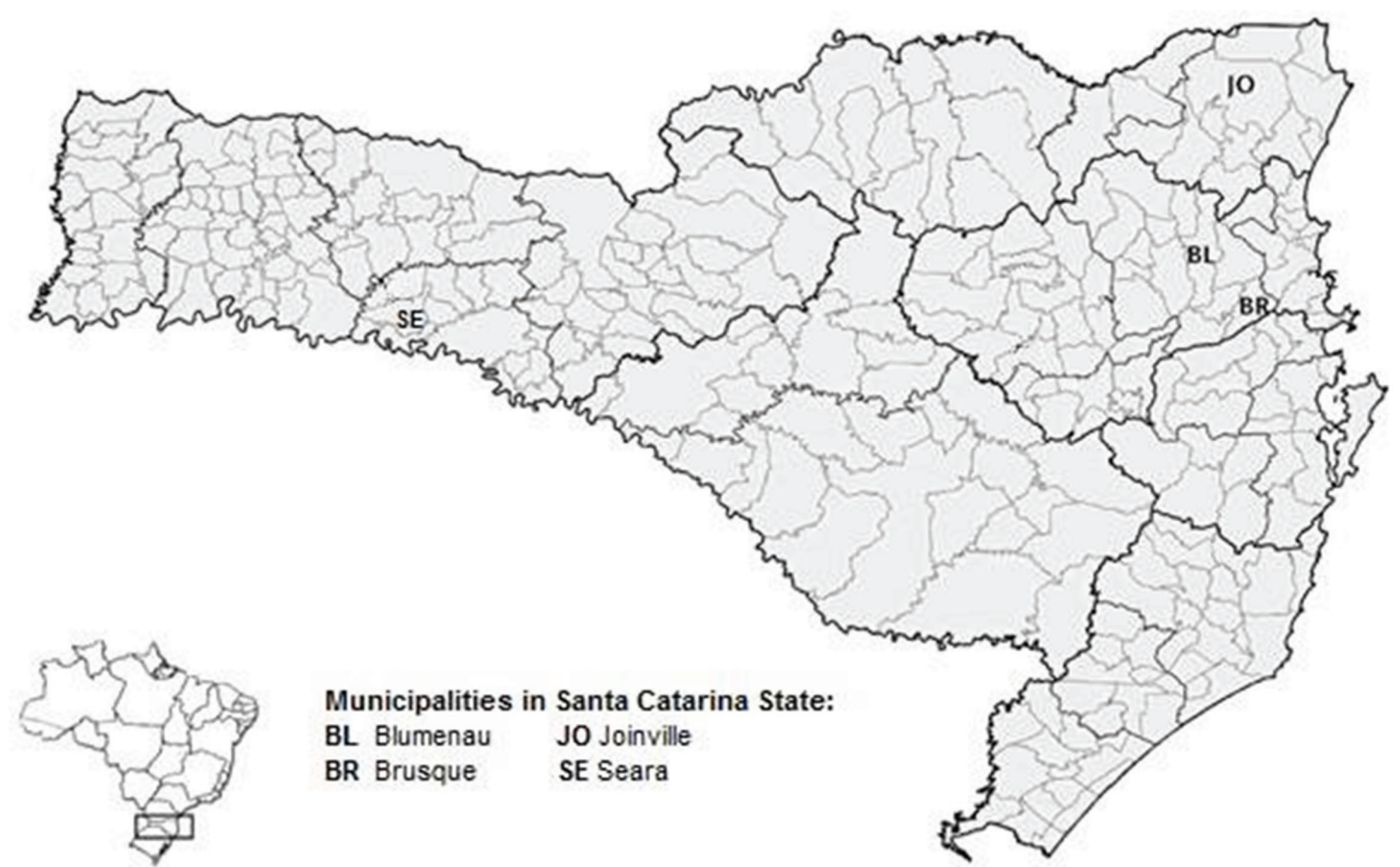

Figura 1. Municípios onde foram realizadas as coletas de Arctiinae por Ceslau Maria Biezanko, Vitor Becker e Fritz Plauman entre os anos 1943 e 1976 no Estado de Santa Catarina, Brasil.

Tabela 1. Coordenadas geográficas, altitude (m), clima (Koppen) e tipo de vegetação dos municípios que apresentaram registros de Arctiinae para o Estado de Santa Catarina, Brasil. (FOD) Floresta Ombrófica Densa. (FOM) Floresta Ombrófila Mista. (Cwa) Clima subtropical seco. (Cfa) Clima subtropical úmido.

\begin{tabular}{|c|c|c|c|c|}
\hline Município & Coordenadas & Altitude & Clima & Vegetação \\
\hline Blumenau & $26^{\circ} 55^{\prime}$ o $8^{\prime \prime} \mathrm{S}$ e $49^{\circ}$ o3' $58^{\prime \prime} \mathrm{W}$ & 21 & Cwa & FOD \\
\hline Brusque & $27^{\circ} 05^{\prime} 53^{\prime \prime} \mathrm{S}$ e $48^{\circ} 55^{\prime} \mathrm{o} 1^{\prime \prime} \mathrm{W}$ & 36 & Cwa & FOD \\
\hline Joinville & $26^{\circ} 18^{\prime} 14^{\prime \prime}$ S e $48^{\circ} 50^{\prime} 42^{\prime \prime} \mathrm{W}$ & 3 & Cwa & FOD \\
\hline Seara & $27^{\circ} 08^{\prime} 56^{\prime \prime}$ S e $52^{\circ} 18^{\prime} 36^{\prime \prime} \mathrm{W}$ & 550 & $\mathrm{Cfa}$ & FOM \\
\hline
\end{tabular}

Dysschema nigrivenata (Hering, 1925); Elysius discoplaga (Walker, 1856); Eucereon capsica (Schaus, 1896); Hypercompe dognini Rothschild, 1910; Hypercompe abdominalis (Walker, 1865); Leucanopsis joasa (Schaus, 1941); Lophocampa dinora (Schaus, 1924); Melese binotata (Walker, 1856); Philoros affinis (Rothchild, 1912); Pseudosphex polybia Kaye, 1911 e Robinsonia praphaea Dognin, 1906.

Recentemente foi elaborada uma lista de espécies ocorrentes em Santa Catarina a partir de dados de espécimes depositados em diversas coleções brasileiras, são referidas 499 espécies (FERRo et al. 2012). Acrescentando os novos registros do presente estudo, o estado passa a apresentar 514 espécies. A riquesa de espécies encontrada através da união destes trabalhos corresponde a $9 \%$ das espécies de Arctiinae neotropicais, cálculo baseado nos dados de HEPPNER (1991) e 26\% da fauna estimada para o Brasil por BROWN JR. \& FreITAS (1999).
A riqueza de mariposas Arctiinae relacionada ao Estado de Santa Catarina está acima da registrada para o Rio Grande do Sul, o qual apresenta um elevado número de estudos. Para esta região foram encontradas aproximadamente 379 espécies (Teston \& Corseuil 2002, 2003a, 2003b, 2004; Ferro \& Teston 2009). Esta discrepancia entre os valores de riqueza descritos para cada estado, em relação aos estudos realizados, pode estar relacionada principalmente ao tipo de ambiente e ao interesse particular de pesquisadores, são necessários mais estudos para esta inferência.

Ainda assim, Ferro et al. (2012) descreve Santa Catarina como uma região consideravelmente subamostrada com relação às mariposas Arctiinae, baseia-se na alta porcentagem de espécies raras com relação à poucos municípios estudados no estado, com baixo número amostral em cada um deles, além da escassez de inventários recentes. Com investimento em pesquisas voltadas a solucionar estas lacunas é possível que novas espécies sejam

Tabela 2. Lista das espécies de Arctiinae registradas para o Estado de Santa Catarina, Brasil. (S) número de espécies. ${ }^{*}$ ) novo registro para o município. $(* *)$ novo registro para o estado.

\section{Táxon} Município(s) 
Tabela 2. Continuação...

Táxon

Município(s)

o6 Argyroeides nephelophora Hampson, 1914

Seara

07 Bertholdia grisescens Rothschild, 1909

Blumenau

o8 Bertholdia pseudofumida Travassos, 1950

Blumenau*, Brusque

o9 Bertholdia soror Dyar, 1901

Seara***

10 Carales astur Cramer, 1777

11 Castrica phalaenoides Drury, 1773

Joinville

12 Cissura decora Walker, 1854

13 Cosmosoma centralis (Walker, 1854)

Brusque*

Brusque

Joinville

14 Cosmosoma ignidorsia Hampson, 1898

Blumenau $^{* * *}$

15 Cosmosoma pellucida Lathy, 1899

16 Cosmosoma sp. 1

17 Ctenucha mortia Schaus, 1901

Seara

18 Demolis albicostata Hampson, 1901

Blumenau*

Blumenau*

19 Dycladia lucetius (Stoll, 1781)

Seara*

Seara

20 Dysschema amphissa Geyer, 1832

Blumenau*, Joinville

21 Dysschema fantasma Butler, 1873

22 Dysschema hilarina Weymer, 1914

Seara

23 Dysschema lucifer Butler, 1873

24 Dysschema neda Klug, 1836

25 Dysschema nigrivenata Hering, 1925

26 Dysschema sacrifica Hübner, [1831]

27 Dysschema subapicalis Walker, 1854

28 Elysius discoplaga Walker, 1856

Seara

Blumenau**

Blumenau*

Seara**

Blumenau*, Joinville

Blumenau*, Brusque*

29 Elysius ordinaria Schaus, 1894

Blumenau***

$30 \quad$ Elysius pyrosticta Hampson, 1905

Seara*

Seara

$31 \quad$ Epidesma ursula (Stoll, 1781)

Blumenau*

32 Eucereon apicalis (Walker, 1856)

Blumenau*

33 Eucereon arenosum Butler, 1877

Blumenau*

34 Eucereon capsica (Schaus, 1896)

Seara $^{* *}$

35 Eucereon discolor (Walker, 1856)

36 Eucereon ladas Schaus, 1892

37 Eucereon quadricolor (Walker, 1855)

Seara*

Blumenau*

Blumenau*

38 Eucereon striatum Druce, 1889

Blumenau*

39 Euceriodes sp. 1

40 Halysidota cinctipes Grote, [1866]

Seara*

41 Halysidota striata Jones, 1908

Blumenau

$42 \quad$ Holophaea erharda Schaus, 1927

Seara*

Seara

43 Horama panthalon viridifusa Schaus, 1904

Seara*

44 Horama sp. 1

Seara*

$45 \quad$ Hyaleucerea sp. 1

46 Hyalurga fenestrata (Walker, 1855)

Seara*

$47 \quad$ Hypercompe abdominalis (Walker, 1865)

48 Hypercompe brasiliensis (Oberthür, 1881)

49 Hypercompe dognini Rothschild, 1910

$50 \quad$ Hypercompe kinkelini Burmeister, 1880

$51 \quad$ Idalus lineosus Walker, 1869

52 Ischnocampa lugubris Schaus, 1892

53 Lepidozikania cinerascens Walker, 1855

54 Leucanopsis joasa Schaus, 1941

55 Leucanopsis leucanina Felder \& Rogenhofer, 1874

56 Leucanopsis sablona Schaus, 1896 
abela 2. Continuação...

\begin{tabular}{|c|c|c|}
\hline & Táxon & Município(s) \\
\hline 57 & Leucanopsis sp. 1 & Blumenau* \\
\hline 58 & Leucanopsis umbrosa Hampson, 1901 & Seara \\
\hline 59 & Lophocampa citrina Sepp, [1852] & Blumenau \\
\hline 60 & Lophocampa dinora Schaus, 1924 & Seara ${ }^{* *}$ \\
\hline 61 & Machadoia xanthosticta Hampson, 1901 & Seara, Blumenau* \\
\hline 62 & Mallodeta clavata Walker, 1854 & Seara* \\
\hline 63 & Mazaeras francki Schaus, 1896 & Blumenau* \\
\hline 64 & Melese binotata Walker, 1856 & Joinville** \\
\hline 65 & Mesothen desperata Walker, 1856 & Seara* $^{*}$ \\
\hline 66 & Mirandisca harpalyce (Schaus, 1892) & Blumenau* \\
\hline 67 & Nelphe confinis (Herrich-Schäffer, 1855) & Seara \\
\hline 68 & Neotrichura nigripes (Heylaerts, 189o) & Brusque* \\
\hline 69 & Neritos repanda Walker, 1855 & Seara \\
\hline 70 & Opharus basalis Walker, 1856 & Blumenau* \\
\hline 71 & Opharus bimaculata Dewitz, 1877 & Seara \\
\hline 72 & Opharus procroides Walker, 1855 & Blumenau* \\
\hline 73 & Ormetica chrysomelas Walker, 1856 & Seara \\
\hline 74 & Ormetica fulgurata Butler, 1876 & Blumenau* \\
\hline 75 & Paracles bilinea Schaus, 1901 & Blumenau* \\
\hline 76 & Paracles fervida Schaus, 1901 & Joinville \\
\hline 77 & Paracles fusca Walker, 1856 & Blumenau \\
\hline 78 & Pareuchaetes aurata Butler, 1875 & Blumenau* \\
\hline 79 & Pelochyta cinerea Walker, 1855 & Blumenau* \\
\hline 80 & Phaegoptera chorima Schaus, 1896 & Seara, Blumenau* \\
\hline 81 & Phaegoptera flavopunctata (Herrich-Schäffer, 1855) & Seara* \\
\hline 82 & Phaegoptera granifera Schaus, 1892 & Blumenau, Joinville \\
\hline 83 & Phaegoptera histrionica Herrich-Schäffer, [1853] & Blumenau* $^{*}$ Joinville* \\
\hline 84 & Phaegoptera schaefferi Schaus, 1892 & Seara* \\
\hline 85 & Philoros affinis (Rothchild, 1912) & Blumenau ${ }^{* *}$ \\
\hline 86 & Pseudohyaleucerea vulnerata (Butler, 1875) & Seara \\
\hline 87 & Pseudosphex polybia Kaye, 1911 & Seara ${ }^{* *}$ \\
\hline 88 & Pseudosphex sp. 1 & Seara* $^{*}$ \\
\hline 89 & Ptychotricos elongatus Schaus, 1906 & Blumenau* \\
\hline 90 & Rhipha subflammans (Rothschold, 1909) & Seara \\
\hline 91 & Robinsonia praphaea Dognin, 1906 & Blumenau** \\
\hline 92 & Romualdia elongata Felder, 1874 & Blumenau*, Joinville \\
\hline 93 & Romualdia opharina Schaus, 1921 & Blumenau*, Joinville \\
\hline 94 & Sthenognatha gentilis Felder, 1874 & Blumenau*, Joinville \\
\hline 95 & Sychesia dryas Cramer, 1775 & Seara* $^{*}$ \\
\hline 96 & Symphlebia catenata Schaus, 1905 & Blumenau* $^{*}$ \\
\hline 97 & Tricypha sp. 1 & Seara* $^{*}$ \\
\hline 98 & Virbia divisa Walker, 1864 & Blumenau* \\
\hline 99 & Viviennea dolens Druce, 1904 & Seara \\
\hline 100 & Viviennea flavicincta Herrich-Schäffer, [1855] & Blumenau*, Joinville \\
\hline 101 & Viviennea salma Druce, 1896 & Brusque \\
\hline
\end{tabular}

descobertas, aumentando a riqueza de Arctiinae catalogada.

\section{REFERÊNCIAS}

Brown Jr., K.S. \& A.V.L. Freitas, 1999. Lepidoptera, p. 225-243. In: Brandão, C.R.F. \& E.M. Cancello (Eds.). Biodiversidade do Estado de São Paulo: síntese do conhecimento ao final do século XX. Invertebrados terrestres. São Paulo, Fapesp, 279p.

Ferro, V.G. \& H.P. Romanowski, 2012. Diversity and composition of tiger moths (Lepidoptera: Arctiidae) in an area of Atlantic Forest in southern Brazil: is the fauna more diverse in the grassland or in the forest? Sociedade Brasileira de Zoologia, 29: 07-18.

Ferro, V.G. \& I.R. Diniz, 2007. Arctiidae (Insecta: Lepidoptera) da Estação Biológica de Boracéia (Salesópolis, São Paulo, 
Brasil). Biota Neotropica, 7: 331-338.

Ferro, V.G. \& J.A. Teston, 2009. Composição de espécies de Arctiidae (Lepidoptera) no Sul do Brasil: relação entre tipos de vegetação e entre a configuração espacial do hábitat. Revista Brasileira de Entomologia, 53: 278-286.

Ferro, V.G., I.M.H. Resende \& M. Duarte, 2012. Mariposas Arctiinae (Lepidoptera: Erebidae) do estado de Santa Catarina, Brasil. Biota Neotropica, 12: 01-15.

Freitas, A.V.L., R.B. Francini \& K.S. Brown Jr., 2006. Insetos como Indicadores Ambientais, p. 125-151. In: Cullen JR., L., R. Rudran, \& C. Valladares-Padua (Eds.). Métodos de estudos em biologia da conservação e manejo da vida silvestre. Curitiba, UFPR, 652p.

Hampson, G.F., 1898. Catalogue of the Lepidoptera Phalaenae in the British Museum. Order of the Trustees, London, 1: XVII.

Hampson, G.F., 1900. Catalogue of the Lepidoptera Phalaenae in the British Museum. Order of the Trustees, London, 2: XVIIIXXXV.

Hampson, G.F., 1901. Catalogue of the Lepidoptera Phalaenae in the British Museum. Order of the Trustees, London, 3: XXXV-LIV.

Hampson, G.F., 1915. Catalogue of the Lepidoptera Phalaenae in the British Museum. Order of the Trustees, London, Supplement 1: XLI.

Hampson, G.F., 1920. Catalogue of the Lepidoptera Phalaenae in the British Museum. Order of the Trustees, London, 2: LXXI.

Heppner, J.B., 1991. Faunal regions and the diversity of Lepidoptera. Tropical Lepidoptera, 2: 01-85.

Hilt, N. \& K. Fiedler, 2006. Arctiid moth ensembles along a successional gradient in the Ecuadorian montane rain forest zone: how different are subfamilies and tribes? Journal of Biogeografy, 33: 108-120.

Hilty, J. \& A. Merenlender, 200o. Faunal indicator taxa selection for monitoring ecosystem health. Biological Conservation, 92: $185-197$.

Seitz, A., 1907. II Abteilung: Die Gross-Schmetterlinge des Amerikanischen Faunengebietes. Band 7: Eulenartige Nachtfalter. VI: 09-67.
Teston, J.A. \& E. Corseuil, 2002. Arctiinae (Lepidoptera, Arctiidae) ocorrentes no Rio Grande do Sul, Brasil. Parte I. Pericopini. Biociências, 10: 261-268.

Teston, J.A. \& E. Corseuil, 2003a. Arctiinae (Lepidoptera, Arctiidae) ocorrentes no Rio Grande do Sul, Brasil. Parte II. Arctiini, Callimorphini e Phaegopterini. Biociências, 11: 6980.

Teston, J.A. \& E. Corseuil, 2003b. Arctiinae (Lepidoptera, Arctiidae) ocorrentes no Rio Grande do Sul, Brasil. Parte III. Ctenuchini e Euchromiini. Biociências, 11: 81-90.

Teston, J.A. \& E. Corseuil, 2004. Diversidade de Arctiinae (Lepidoptera, Arctiidae) capturados com armadilha luminosa, em seis comunidades no Rio Grande do Sul, Brasil. Revista Brasileira de Entomologia, 48: 77-90.

Teston, J.A., A. Specht, R.A. Di Mare \& E. Corseuil, 2006. Arctiinae (Lepidoptera, Arctiidae) coletados em unidades de conservação estaduais do Rio Grande do Sul, Brasil. Revista Brasileira de Entomologia, 50: 280-286.

Teston, J.A., A.P. Silveira \& E. Corseuil, 2009. Abundância, Composição e Diversidade de Arctiinae (Lepidoptera, Arctiidae) num fragmento de Mata Atlântica em Iraí, RS, Brasil. Revista Brasileira de Zoociência, 11: 65-72.

Watson, A. \& D.T. Goodger, 1986. Catalogue of the Neotropical Tiger-moths. Occasional Papers on Systematic Entomology, 1: 1-71.

Zahiri, R., I.J. Kitching, J.D. Lafontaine, M. Mutanen, L. Kaila, J.D. Holloway \& N. Wahlberg, 2011. A new molecular phylogeny offers hope for a stable family-level classification of the Noctuoidea (Lepidoptera). Zoologica Scripta, 40: 158173 .

Zahiri, R., J.D. Holloway, I.J. Kitching, J.D. Lafontaine, M. Mutanen \& N. Wahlberg, 2012. Molecular phylogenetics of Erebidae (Lepidoptera, Noctuoidea). Systematic Entomology, 37: 102-124.

\section{Recebido em: 06/o9/2013}

Aceito em: 21/03/2014

\section{Como citar este artigo:}

Silva, J.M. \& E.J.E. Silva, 2014. Contribuição para o Conhecimento da Fauna de Arctiinae (Lepidoptera: Erebidae) no Estado de Santa Catarina, Brasil. EntomoBrasilis, 7 (3): 222-226.

Acessível em: doi:10.12741/ebrasilis.v7i3.387
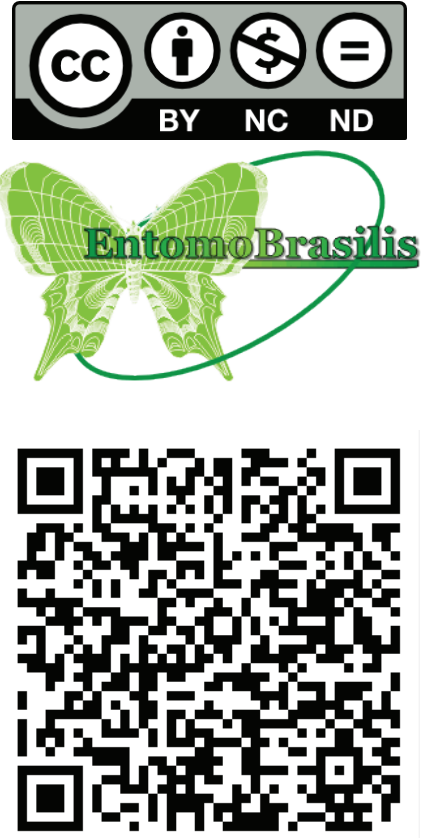